\title{
Gegen den Versuch, das Arzttum zu zerstören
}

\section{Rolf H. Adler}

Prof. em., Dr. med., ehem. Chefarzt Innere Medizin, C.-L.-Lory-Haus, Inselspital Bern

Gegenstand dieses Artikels ist ein Verwaltungsgerichtsurteil in einem Rückforderungsprozess. Darin wird festgehalten, welche Behandlung psychosomatischer Patienten im Rahmen der OKP nicht zu den von einem Arzt zu erbringenden Leistungen zählt. Bei dieser Betrachtung geht es dem Autor nicht um die Klärung der Frage, wie gut der Patient ärztlich versorgt worden ist.

\section{Das Urteil hält fest:}

Es gehört zur Behandlung psychosomatischer Patienten im Rahmen des OKP nicht zu den von einem Arzt zu erbringenden Leistungen:

a) Alltagssorgen zu besprechen;

b) Anteilnahme auszusprechen;

c) Patienten auf deren Wunsch hin zu beraten und zu bedauern;

d) Ansonsten nicht erhältliche Zuwendung zu spenden;

e) Lebensberatung zu gewähren.

(Anmerkung des Autors: Der Einfachheit halber habe ich jede dieser Feststellungen mit den Buchstaben a bis e bezeichnet.)

\section{Zuerst zum Begriff «Psychosomatik»}

Der Begriff ist von Heinroth (1773 bis 1843) eingeführt worden und schon jahrzehntelang obsolet, denn er beruht auf der zu einfachen Vorstellung, psychische

\section{Somatische, psychische und soziale Faktoren wirken ineinander.}

Ursache - somatische Folgen. Seit 1977 und dem Artikel Engels in Science [1] denken und handeln wir als Forscher und Kliniker im biopsychosozialen Konzept: Ein Individuum ist hierarchisch aufgebaut. Die Ebenen reichen vom Molekularen bis ins Soziale. Somatische, psychische und soziale Faktoren wirken ineinander. Dabei geht es um Koppelungen und Entkoppelungen der Faktoren auf den verschiedenen Ebenen und zwischen diesen, mit der Frage, ob Gesundheit erhalten bleibt oder Krankheit entsteht [2, 3]. Die Aufgabe des Arztes besteht darin, die somatischen, psychischen und sozialen Faktoren in ihren Zusammenhängen zu erfassen, zu gewichten und daraus Handlungsanweisungen fürs ärztliche Tun abzuleiten. Schon der Neurochirurg Harvey Cushing (1869-1939), der übrigens bei Theodor Kocher in Bern gearbeitet hat, hielt fest, dass der Arzt nicht nur die Krankheit, sondern den Menschen mit seiner Krankheit, und nicht nur diese beiden, sondern den Menschen mit seiner Krankheit in seiner Umwelt zu erfassen hat. Die Verfasser des Gerichtsurteils hinken also um hundert und mehr Jahre heutigen Erkenntnissen nach.

\section{Zu den Bemerkungen a bis e}

$\mathrm{Zu} a$ und $b$ : Letztes Jahr ruft mich ein Freund und Kollege an. Einer seiner Freunde, 68-jährig, hat seine 69 Jahre alte Frau drei Monate nach der Diagnose Pankreaskarzinom verloren. Mein Freund möchte wissen, welches Medikament er dem Witwer anlässlich der kurz bevorstehenden Beerdigung geben solle, da dieser befürchte, während der Abdankung von Gefühlen überwältigt zu werden und in Ohnmacht zu fallen. Ich antworte, dass Verordnungen per Telefon Gefahren bergen [4], die auch schon zum Tode geführt haben, und dass der Arzt nur Menschen Empfehlungen am Telefon geben dürfe, die er als seine Patienten genau kennt. Ich bitte ihn, seinem Freund zu raten, mich so bald wie möglich anzurufen, um für den nächsten Tag einen Termin zu vereinbaren. Am nächsten Morgen kommt der Betroffene in meine internistische Sprechstunde. Das Gespräch umfasst seine Befürchtungen, seine Gefühle, seine intensive Trauer. Wir besprechen zusammen, dass die Stärke der Trauer der Stärke der 
Liebe zu seiner Frau entspreche und dass ich in meinem Nachttisch ein Zettelchen aufbewahre, auf dem steht, dass Gefühle Tatsachen sind, auch wenn sie «nur» Gefühle sind, wie Friedrich Glauser (1935) einst im Roman Die Fieberkurve geschrieben hat [5]. Als Arzt habe ich damit Psychisches in meine Tätigkeit eingeschlossen. Ich erfahre, dass er einen Sohn und eine Tochter hat, wie seine Beziehungen zu ihnen sind, dass er mit Freunden häufig Bergtouren unternehme und dass sie zur Beerdigung kämen. Einbe-

Ich bin stolz und glücklich, noch ein Arzt zu sein, der biopsychsosozial denkt und handelt.

zug des Sozialen. Er kommt auf den Gedanken, bei der Abdankung eng bei seinen Kindern und seinen Freunden zu sein. Er verzichtet auf ein Medikament. Ich bin auch dafür, denn ich habe mich nach seinem Verhalten anlässlich früherer Verluste erkundigt, die er gut gemeistert hat (psychische Entwicklung). Ich bitte ihn, mir in den nächsten Tagen zu berichten. Es erfolgt seine Mitteilung, es sei gut gegangen, und einige Wochen später schreibt er mir, dass er mit seinen Freunden wieder zu Berge gehe. Ich mache ihn darauf aufmerksam, dass Trauern ein Prozess mit Auf und Ab sei und dass er mich bei Bedarf ruhig anrufen solle [6]. Ich bin stolz und glücklich, noch ein Arzt zu sein, der biopsychsosozial denkt und handelt.

Dabei erinnere ich mich an ein Geschehnis aus dem Jahre 1934, das Engel erwähnt [7]: Ein Lamahengst ist wild geworden, hat sein Gehege durchbrochen. Er kann nicht eingefangen werden und wird erschossen. Seine Gefährtin folgt ihm, steht über dem Toten und bricht tot zusammen! Heute kennen wir solche Abläufe auch beim Menschen, beispielsweise beim sogenannten Takotsubo-Syndrom, bei dem akuter Stress zur Schädigung von Herzmuskelzellen und zum Versagen der Herzpumpfunktion führt [8]. Es ist zudem bekannt, dass Verwitwete nach Partnerverlust während zweier Jahre eine um 40\% erhöhte Mortalität aufweisen [9]. Mit diesen Ausführungen halte ich Punkt b auch für geklärt.

Zu c: Das Problem «Beratung» muss hier besprochen werden. Beispielsweise beim Impfgespräch mit Müttern, die Angst vor dem Impfen haben. Und deren Ängste so gross sind, dass sie eine Impfung ihrer Kinder deshalb ablehnen. Diese Ängste müssen berücksichtigt und thematisiert werden [10]. Denn die Masernimpfung auszulassen kann zu tödlichen Meningoencephalitiden, zu hospitalisationsbdürftigen Pneumonien und zu Otitiden führen.
Zu d: Patienten nach Herzinfarkt, die depressiv sind, weisen ein erhöhtes Risiko auf, einen neuen Infarkt zu erleiden und früher zu sterben [11]. Wer, ausser dem Arzt, vermag eine Depression, die behandlungsbedürftig ist, in einer solchen Situation zu entdecken und korrekt zu behandeln?

$Z u$ e: Aus dem Text des Verwaltungsgerichts geht hervor, dass die verfassenden Juristen nicht wissen, was ärztliche Beratung wirklich bedeutet. Die Patienten sind intelligent genug, um zu wissen, welchem eigenen Rat sie zu folgen hätten. Sie sind aber oft dazu nicht imstande, denn Gefühle wie Angst, Schuld, Wut, Hilflosigkeit, Hoffnungslosigkeit usw., die auftauchen, wenn sie an die Durchführung des eigenen Ratschlags gehen, hindern sie daran. Bei der Beratung muss der Arzt auf diese Gefühle eingehen, denn unbesprochen und nicht mit dem Arzt geteilt, bieten sie dem Befolgen der eigenen Überlegungen Widerstand. Greenson, renommierter Psychoanalytiker, hat das Problem des Ratschlägegebens in Practice and Theory of Psychoanalysis gewürdigt [12].

\section{Überlegungen zur Qualitätsbeurteilung}

Diese spielt ja im erwähnten Urteil eine entscheidende Rolle. Qualität kann leicht beurteilt werden, wenn das zu beurteilende Produkt einfach gestaltet ist, wie eine Coca-Cola-Flasche [13]. Ein Mensch ist aber viel komplexer. Vom Arzt verwendete Zeit und eingesetzte Tests wie Labor-, Röntgen- und weitere Untersuchungen lassen die Qualität nicht beurteilen. Die Beurteilung gelingt nur einigermassen, wenn ein Arzt seine Tätigkeit durch einen erfahrenen Kollegen prüfen lässt. Dies findet statt: Wenn der Assistenzarzt den beim Spitaleintritt untersuchten Patienten seinem Oberarzt oder Chefarzt vorstellt, wenn er am Morgen nach seinem Nachtdienst den versammelten Kollegen über seinen

\section{Unterziehen sich Juristen, Gesundheitsökono-} men, Krankenkassen-Leiter und Politiker solch rigorosen Qualitätssicherungen?

Nachtdienst berichtet, wenn er beim gemeinsamen Röntgenrapport die Bilder seines Patienten vorstellt, auf der Oberarzt- und Chefvisite, wenn er seinen Austrittsbericht an den Hausarzt seinem Oberarzt oder Chef vorlegt. Nach abgeschlossener Aus- und Weiterbildung ist er genötigt, als Fortbildung an Besprechungen mit Kollegen teilzunehmen und Fortbildungsveranstaltungen zu besuchen, und dies zu belegen. Er erhält dafür Punkte. Ende Jahr muss er eine bestimmte Punktzahl vorlegen. Frage: Unterziehen sich Juristen, 
Gesundheitsökonomen, Krankenkassenleiter und Politiker solch rigorosen Qualitätssicherungen? Würden sie, das kann mit Fug und Recht bedacht werden, sich zum Beispiel einem Brückenbauingenieur gegen-

Die Behauptung, Ärzte würden unnötige Untersuchungen veranlassen, um zu verdienen, trifft vielleicht auf einige schwarze Schafe zu.

über erlauben, dessen Stabilitätsabklärungen und Berechnungen in Frage zu stellen? Genau dieser Übergriff wird gegenüber der medizinischen Profession getan eine Anmassung.

\section{Nur vereinzelt schwarze Schafe}

Die so oft gehörte Behauptung, Ärzte würden unnötige Untersuchungen veranlassen, um zu verdienen, trifft vielleicht auf einige schwarze Schafe der Profession zu, wie sie in allen Berufsgruppen vorkommen. Es gibt aber nicht Herden von schwarzen Schafen, wie sie uns Gesundheitsökonomen, Politiker und Krankenkassen glauben machen wollen. Es ist für uns Ärzte ein erhabenes und uns schon als Studenten vorgelebtes Anliegen, mit möglichst wenig Aufwand Patienten abzuklären und zu behandeln. Unsicherheit und Angst und Übermittlungspannen von Daten mögen hie und da zu

Abklärungen führen, die im Rückblick fragwürdig bis überflüssig erscheinen. Im Rückblick ist man immer gescheiter.

\section{Fazit}

Somit komme ich zum Schluss: Liebe Kolleginnen und Kollegen, wehren Sie sich mit mir zusammen gegen die ungerechtfertigten Einmischungen in die Medizin, die zuletzt unsere Patienten auszulöffeln haben. Halten Sie sich an Major Cavaluzzi in Carl Spittelers (1845-1924) Gedicht Die jodelnden Schildwachen: «Komm her beim Styx, stoss an beim Eid, wer da nicht mitmöggt, tut mir leid.»

Literatur

1 Engel GL. The need for a new medical model: a challenge for biomedicine. Science. 1977;196:129-36.

2 Von Uexküll T. Psychosomatische Medizin. Urban und Fischer: München und Jena; 2010, 1. Kap.

3 Adler RH. Engels biopsychosoziales Konzept ist auch heute noch bedeutsam. In: Herausforderung für die Biomedizin: Das biopsychosoziale Konzept. EMH, Schweiz Ärzteverlag 2017; S.9-18. Erstpubl. J Psychosom Res. 2009;607-11.

4 Adler RH. TelMed: gefährlich, unwirtschaftlich und entbehrlich. Schweiz Med Forum. 2012;12:679-80.

5 Friedrich Glauser. Die Fieberkurve (1935).

6 Engel GL. Is grief a disease? A challenge for medical research. Psychosom Med. 1961;23:18-22.

7 Engel GL. Sudden and rapid death during psychological stress. Folklore or folkwisdom? Ann Intern Med. 1971;74:771-82.

8 Sato H, Tateishi H, Ushida T, et al. Myocardial stunning due to simultaneous multiple vessel coronary spasms. J Cardiol. 1991;21:203-14.

9 Parker CM, Benjamin B, Fitzgerald GL. Broken heart: a statistical analysis of increased mortality among widowers. BMJ. 1969;1:740-3.

10 Peters N. Impfung in der Praxis. Prim Hosp Care. 2018;18:118-21.

11 Ziegelstein RC. Depression in patients recovering from myocardial infarction. JAMA. 2011;286:1621-7.
12 Greenson RR. The technique and practice of psychoanalysis I. International Universities Press, New York 1967.

13 Adler RH. Qualitätssicherung und Vernetzung. Praxis. 2012;101:781-5 